Original Research

\title{
Improvement of depiction of the intracranial arteries on brain CT angiography using deep learning reconstruction
}

\author{
Chuluunbaatar Otgonbaatar ${ }^{1}$, Jae-Kyun Ryu ${ }^{2}$, Seonkyu Kim ${ }^{2}$, Jung Wook Seo ${ }^{3}$, Hackjoon Shim ${ }^{4, *}$, Dae Hyun Hwang ${ }^{5, *}$ \\ ${ }^{1}$ Department of Radiology, College of Medicine, Seoul National University, 03080 Seoul, Republic of Korea \\ ${ }^{2}$ Medical Imaging Al Research Center, Canon Medical Systems Korea, 06173 Seoul, Republic of Korea \\ ${ }^{3}$ Department of Radiology, Inje University Ilsan Paik Hospital, 10380 Goyang, Republic of Korea \\ ${ }^{4}$ Connect Al Research Center, Yonsei University College of Medicine, 03772 Seoul, Republic of Korea \\ ${ }^{5}$ Department of Radiology, Inje University Seoul Paik Hospital, 04551 Seoul, Republic of Korea \\ *Correspondence: hjshim@yuhs.ac (Hackjoon Shim); mddhhwang@naver.com (Dae Hyun Hwang)
}

DOI:10.31083/j.jin2004097

This is an open access article under the CC BY 4.0 license (https://creativecommons.org/licenses/by/4.0/).

Submitted: 25 October 2021 Revised: 22 November 2021 Accepted: 1 December 2021 Published: 30 December 2021

To evaluate the ability of a commercialized deep learning reconstruction technique to depict intracranial vessels on the brain computed tomography angiography and compare the image quality with filtered-back-projection and hybrid iterative reconstruction in terms of objective and subjective measures. Forty-three patients underwent brain computed tomography angiography, and images were reconstructed using three algorithms: filtered-back-projection, hybrid iterative reconstruction, and deep learning reconstruction. The image noise, computed tomography attenuation value, signal-to-noise ratio, and contrast-to-noise ratio were measured in the bilateral cavernous segment of the internal carotid artery, vertebral artery, basilar apex, horizontal segment of the middle cerebral artery and used for the objective assessment of the image quality among the three different reconstructions. The subjective image quality score was significantly higher for the deep learning reconstruction than hybrid iterative reconstruction and filtered-back-projection images. The deep learning reconstruction markedly improved the reduction of blooming artifacts in surgical clips and coiled aneurysms. The deep learning reconstruction method generally improves the image quality of brain computed tomography angiography in terms of objective measurement and subjective grading compared with filteredback-projection and hybrid iterative reconstruction. Especially, deep learning reconstruction is deemed advantageous for better depiction of small vessels compared to filtered-back projection and hybrid iterative reconstruction.

\section{Keywords}

Computed tomography; Brain angiography; Intracranial vessel; Image reconstruction; Deep learning reconstruction algorithm

\section{Introduction}

Brain multidetector-row Computed Tomography Angiography (CTA) is a suitable noninvasive imaging modality frequently used in cases of vascular diseases including aneurysm, vessel dissection, vascular malformations, stroke, and tumors [1]. The technical improvements with high spatial and temporal resolution enabled CTA to produce comparable image quality to conventional angiography and evaluate cerebrovascular diseases, especially intracranial aneurysms [2]. However, lowering the tube voltage and currents results in a reduction of radiation exposure, which causes for degradation of image quality on CTA and affects its diagnostic accuracy. Especially the detection of small diameter vessels by CTA still presents a challenge. To visualize the intracranial vessels clearly and accurately, there should be a great deal to improve the image quality by developing image reconstruction algorithms.

Initially, hybrid iterative reconstruction (Hybrid IR) and model-based iterative reconstruction (MBIR) were effective until deep learning image reconstruction appeared on the stage. Several studies [3-5] reported that MBIR improves the delineation of small vascular structures with high image quality and spatial resolution compared to Hybrid IR. However, long reconstruction time limits its routine use in clinical practice. Clinical studies have confirmed that the low radiation dose used during hybrid iterative reconstruction and deep learning reconstruction (DLR) implies patient safety while improving image quality; additionally, lower image noise, higher contrast-to-noise ratio (CNR), and lower blooming artifacts were observed [6-9].

Advancements in developing deep learning reconstruction have led to better outcomes by low radiation exposure and excellent imaging quality. The Food and Drug Administration has approved two artificial intelligent image reconstruction algorithms for clinical purposes: Advanced Intelligent Clear-IQ Engine (AiCE, Canon Medical Systems Corporation, Otawara, Japan) [10] and TrueFidelity ${ }^{T M}$ system (GE Healthcare, Waukesha, WI) [11]. By utilizing deep convolutional neural networks, AiCE learns differentiation between the signal and the noise with the target images of MBIR images (FIRST, forward projected model-based iterative reconstruction, Canon Medical Systems Corporation, Otawara, Japan) (Fig. 1). On the other hand, the TrueFidelity ${ }^{T M}$ system is trained with high-dose filtered-back projection (FBP) images [12]. 


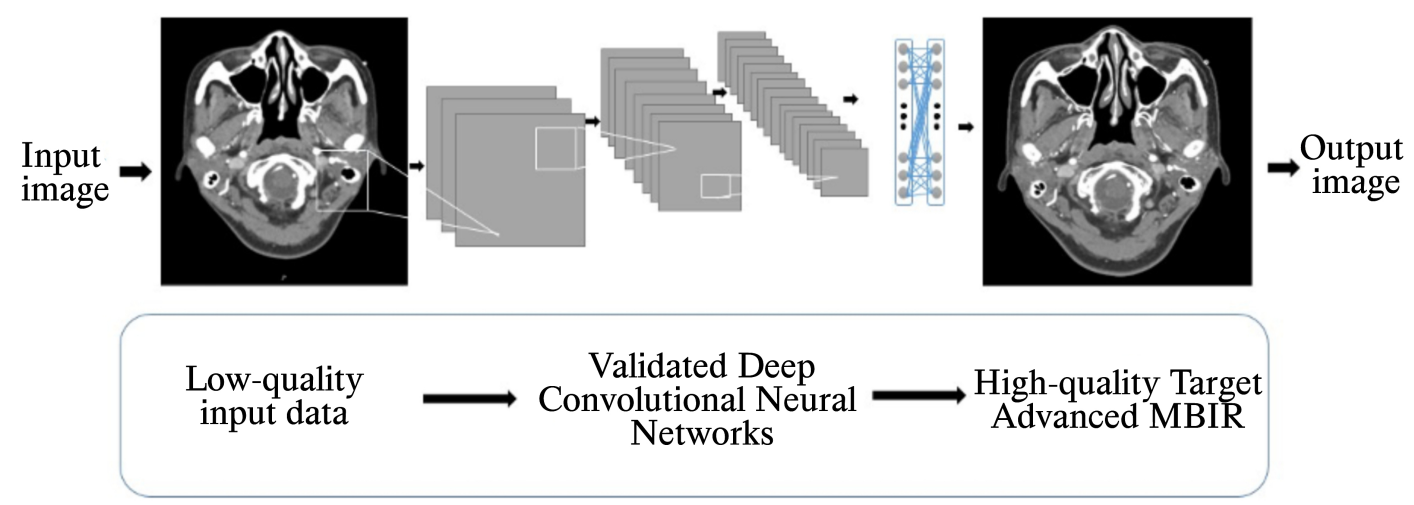

Fig. 1. Overview of deep learning reconstruction. The vast learning capacities of the deep convolutional neural network allow for differentiation between the signal and the noise. Consequently, deep learning reconstruction (DLR) reduces artifacts and noise separately from the signal. AiCE, a DLR used in this study, was trained to use advanced model-based iterative reconstruction (MBIR) target images.

Several studies have utilized DLR in the abdominal, chest, and brain CT imaging and cardiopulmonary CTA and found better image quality than other image reconstruction algorithms [13-18]. No studies so far have investigated the application of AiCE to brain CTA protocols. Therefore, we propose to evaluate the ability of a commercialized deep learning reconstruction technique (AiCE) to depict intracranial vessels on the brain CTA and compare the image quality with other reconstruction algorithms (FBP and Hybrid IR) in terms of objective and subjective measures.

\section{Methods \\ 2.1 Radiation dose}

The dose-length product (DLP) and CT dose indexvolume (CTDIvol) values were collected from the dose reports.

\subsection{Patients}

A total of 43 consecutive patients scheduled for brain CTA from October 2020 to November 2020 were included in this study. This retrospective study was approved by the Institutional Review Board. The exclusion criteria were as follows pregnancy, allergy to iodinated contrast, and severe renal disease.

\subsection{CT scanning protocols and image reconstruction}

All CT examinations were performed using a 320 multidetector row scanner (Aquillion ONE PRISM version 10.4, Canon Medical System, Otawara, Japan) with a protocol consisting of a tube voltage of $120 \mathrm{kVp}$, tube current of $150 \mathrm{mAs}$, field of view of $220 \mathrm{~mm}$, detector collimation of $80 \times 0.5$ $\mathrm{mm}, 0.5$ second gantry rotation time, $0.5 \mathrm{~mm}$ slice thickness, and helical scanning. The slice interval was $0.25 \mathrm{~mm}$ implemented by the double slice technique. Each section is 110 $\mathrm{mm}$ long in the longitudinal direction. The scan was triggered using the automatic bolus-tracking program (Surestart, Canon Medical Systems Corporation, Otawara, Japan) in the common carotid artery at the level of the C4 vertebra (trig- ger threshold was set $150 \mathrm{HU})$. A total of $90 \mathrm{~mL}$ of non-ionic contrast agent (Iopamidol, Pamiray 370, Dongkook, Korea) was administered in the antecubital vein at a rate of $4 \mathrm{~mL} / \mathrm{sec}$ followed by $90 \mathrm{~mL}$ saline at a rate of $3.8 \mathrm{~mL} / \mathrm{sec}$ using an ulrich CT motion Injector (CT motion, ulrich Medical, Ulm, Germany).

Images were reconstructed using three different algorithms: filtered-back projection (FBP) with FC23 kernel, hybrid iterative reconstruction (Adaptive Iterative Dose Reduction 3-D, AIDR-3D, Canon Medical Systems Corporation, Otawara, Japan) with FC23 standard kernel, and deep learning reconstruction (Advanced Intelligent Clear IQ Engine, AiCE, Canon Medical Systems Corporation, Otawara, Japan) with brain CTA standard option. The CTA images were sent to workstation (Vitrea version 7.12, Vital, Minneapolis, USA) for analysis.

\subsection{CT image analysis \\ 2.4.1 Objective image analysis}

Image noise, CT attenuation value (HU), Signal-to-noise ratio (SNR), and Contrast-to-noise ratio were used to objectively assess the CTA images reconstructed by FBP Hybrid IR and DLR.

Image noise was derived from the standard deviation of the HU by placing the largest possible seven regions of interests (ROIs) in the center of the vessel depending on the diameter of each vessel, i.e., the right and left cavernous segments of the internal carotid artery (ICA), right and left vertebral artery, basilar apex, and the right and left M1 segment of the middle cerebral artery (MCA), avoiding the inclusion of the vessel wall (Fig. 2). As the reference for contrast, another ROI $\left(20 \mathrm{~mm}^{2}\right)$ was placed in the right or left of the thalamic gray matter at the level of each basal ganglia.

The SNR was assessed by dividing the attenuation of the vessels by its standard deviation. These vessels include the right and left cavernous segment of the ICA, right and left vertebral artery, basilar apex, and right and left M1 segments of the MCA. 


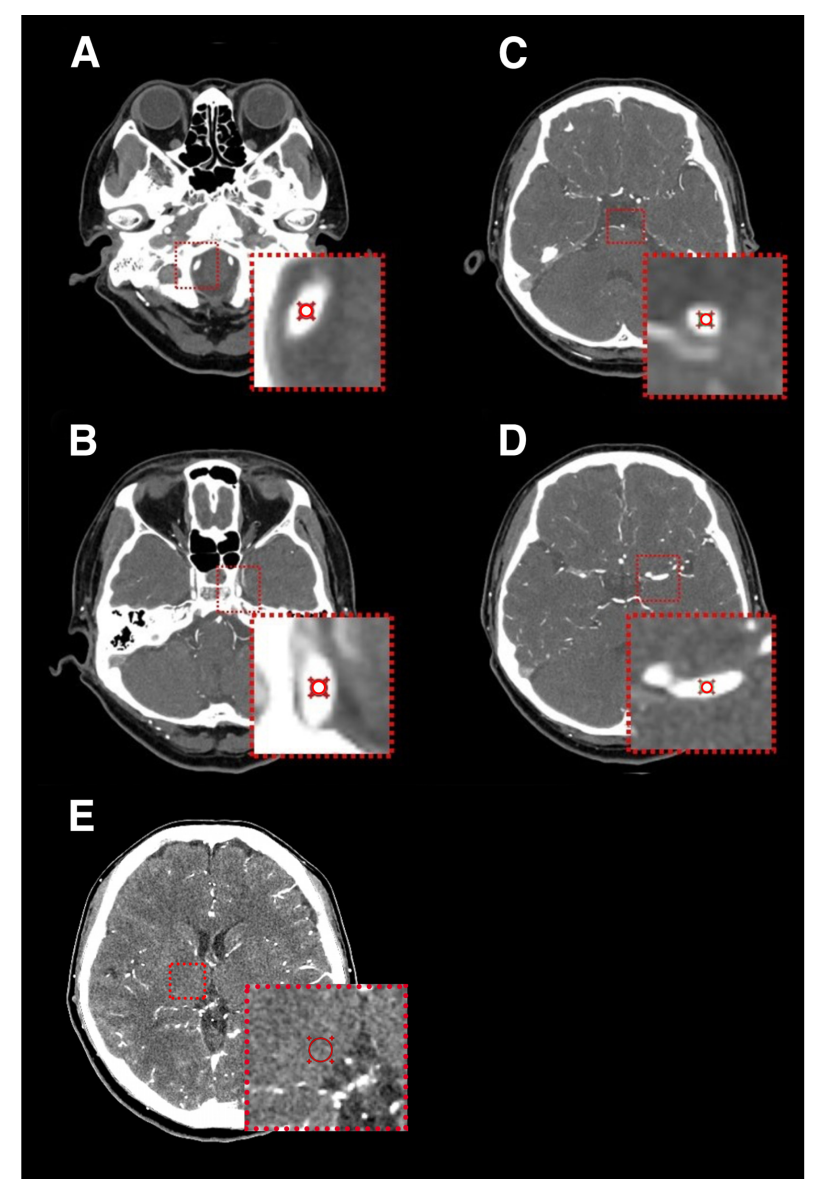

Fig. 2. Measurements of objective image quality in the brain CTA. The region of interest (ROI) was placed in the center of the right and left vertebral artery (A), basilar apex (B), right and left cavernous segment of the internal carotid artery (C), right and left M1 segments of the middle cerebral artery (D), and right thalamic gray matter (E) avoiding the inclusion of a vessel wall at the same position in the three image reconstructions with DLR, Hybrid IR, and FBP.

The CNR was measured as the contrast divided by the noise strength. The contrast was difference between CT attenuation of the vessels and the attenuation of the thalamic gray matter. The vessels include the right and left cavernous segments of the ICA, right and left vertebral arteries, basilar apex, and right and left M1 segments of the MCA.

The surgical clips' sharpness and aneurysm coils were assigned with their perimeter and 10-90\% edge rise distance (ERD) on FBP, Hybrid IR, and DLR. The perimeter of surgical clips and aneurysm coils was analyzed by manual placement of an electronic caliper on the workstation (Vitrea, Vital, Minneapolis, USA). On axial images, the CT attenuation profile with a horizontal line through the center of the clip and coil was determined in the same location for all reconstruction methods. The 10-90\% ERD was considered the distance of $10 \%$ and the $90 \%$ of the peak CT attenuation number. The mean value of three measurements was used for analysis. A shorter edge rise distance demonstrates a higher sharpness. To assess the sharpness of the surgical clips and aneurysm coils, normalized profile curves were compared in the images reconstructed with FBP, Hybrid IR, and DLR using ImageJ software (version 3.0, LOCI, University of Wisconsin, Madison, Wisconsin, USA) [19].

\subsubsection{Subjective image analysis}

The images were subjectively evaluated by two neuroradiologists (each with reading experience longer than five years). The neuroradiologists were blinded to the image reconstruction methods and randomly evaluated the CTA images. A five-point Likert scale was used for the analysis: $5=$ excellent image quality, exquisite vessel delineation wall with barely perceived image noise; 4 = good image quality, good vessel delineation wall with minimal image noise; 3 = acceptable image quality, moderate vessel delineation wall with moderate image noise; 2 = suboptimal image quality, fair vessel sharpness and vessel delineation wall with severe image noise; and 1 = poor limited image quality, limitation in the vessel wall delineation with excessive image noise.

\subsection{Statistical analysis}

Continuous variables were shown as mean \pm standard deviation. The Kruskal Wallis test was used to compare the image noise, CT attenuations, SNR, CNR, and ERD between the image reconstructions. Interobserver agreement was assessed with the Cohen kappa $\kappa$ coefficient where a $\kappa$ value of less than 0.2 signified poor; 0.21-0.4, fair; 0.41-0.6, moderate; 0.61-0.8, good; and 0.81-1 excellent agreement [20]. Statistical significance was set at $p<0.05$. Statistical analysis was performed using the SPSS statistical software ver 25.0 (IBM, Armonk NY, USA).

\section{Results \\ 3.1 Radiation dose}

The mean DLP was $219.05 \pm 20.25 \mathrm{mGy} \bullet \mathrm{cm}$, and the mean CTDIvol was $19.30 \mathrm{mGy}$.

\subsection{Demographic characteristics}

Forty-three patients were included in the study (19 were male $(55.8 \%)$, and 24 were female $(44.2 \%))$. Their mean age was $56.77 \pm 16.31$ ranging from $15-88$ years. The indications for follow-up brain CTAs were as follows: intracranial aneurysm $(n=8)$, vessel dissection $(n=2)$, trauma $(n=1)$, hemorrhage $(n=6)$, acute ischemic stroke $(n=2)$, follow-up study of old infarct $(n=5)$, and normal findings $(n=12)$.

\subsection{Objective image quality analysis}

The objective analysis findings of the FBP, Hybrid IR, and DLR are shown in Tables 1,2. In all patients, the average noise was $19.80 \pm 5.57,16.51 \pm 6.00$, and $10.95 \pm 3.77$ in the FBP, Hybrid IR, and DLR analysis, respectively $(p<0.001)$. The noise reduction resulting from using DLR was approximately $33.68 \%$ and $44.70 \%$ compared with Hybrid IR and FBP.

There was no significant difference in the attenuation of the thalamus and right and left cavernous segments of the ICA among all image reconstruction methods. On the other 
Table 1. Results of image noise and attenuation.

\begin{tabular}{|c|c|c|c|c|c|c|c|}
\hline & \multicolumn{3}{|c|}{ The mean value $\pm S D$} & \multicolumn{4}{|c|}{$p$-value } \\
\hline & FBP & Hybrid IR & DLR & $\begin{array}{c}\text { FBP vs Hybrid IR } \\
\text { vs DLR }\end{array}$ & $\begin{array}{c}\text { FBP vs Hybrid } \\
\text { IR }\end{array}$ & FBP vs DLR & $\begin{array}{c}\text { Hybrid IR vs } \\
\text { DLR }\end{array}$ \\
\hline \multicolumn{8}{|c|}{ Image noise (HU) } \\
\hline Thalamus & $14.71 \pm 1.60$ & $9.92 \pm 0.95$ & $9.00 \pm 0.77$ & 0.001 & 0.001 & 0.001 & 0.001 \\
\hline ICA right & $19.16 \pm 4.37$ & $15.89 \pm 5.30$ & $11.16 \pm 3.26$ & 0.001 & 0.001 & 0.001 & 0.001 \\
\hline ICA left & $19.71 \pm 4.34$ & $16.87 \pm 4.64$ & $11.87 \pm 3.25$ & 0.001 & 0.001 & 0.001 & 0.001 \\
\hline VA right & $19.87 \pm 3.25$ & $16.32 \pm 4.72$ & $9.65 \pm 2.70$ & 0.001 & 0.001 & 0.001 & 0.001 \\
\hline VA left & $19.26 \pm 4.32$ & $16.03 \pm 3.85$ & $10.09 \pm 2.85$ & 0.001 & 0.001 & 0.001 & 0.001 \\
\hline BA apex & $20.73 \pm 6.15$ & $17.85 \pm 6.26$ & $11.23 \pm 4.29$ & 0.001 & 0.001 & 0.001 & 0.001 \\
\hline MCA right & $23.37 \pm 7.24$ & $20.14 \pm 7.86$ & $12.59 \pm 5.50$ & 0.001 & 0.001 & 0.001 & 0.001 \\
\hline MCA left & $22.18 \pm 5.72$ & $19.04 \pm 6.18$ & $12.03 \pm 4.37$ & 0.001 & 0.001 & 0.001 & 0.001 \\
\hline Average & $19.80 \pm 5.57$ & $16.51 \pm 6.00$ & $10.95 \pm 3.77$ & 0.001 & 0.001 & 0.001 & 0.001 \\
\hline \multicolumn{8}{|c|}{$\mathrm{CT}$ attenuation $(\mathrm{HU})$} \\
\hline Thalamus & $39.20 \pm 3.78$ & $39.45 \pm 2.90$ & $38.64 \pm 2.53$ & 0.289 & 0.006 & 0.003 & 0.005 \\
\hline ICA right & $329.03 \pm 84.19$ & $327.11 \pm 83.97$ & $338.27 \pm 87.07$ & 0.743 & 0.001 & 0.001 & 0.001 \\
\hline ICA left & $333.85 \pm 85.15$ & $336.22 \pm 93.83$ & $343.99 \pm 94.60$ & 0.721 & 0.008 & 0.002 & 0.001 \\
\hline VA right & $264.01 \pm 71.03$ & $260.85 \pm 68.18$ & $331.90 \pm 162.75$ & 0.004 & 0.002 & 0.001 & 0.001 \\
\hline VA left & $271.86 \pm 76.11$ & $269.37 \pm 71.03$ & $314.66 \pm 83.60$ & 0.010 & 0.001 & 0.001 & 0.001 \\
\hline BA apex & $258.07 \pm 65.49$ & $242.03 \pm 66.44$ & $310.02 \pm 81.32$ & 0.001 & 0.001 & 0.001 & 0.001 \\
\hline MCA right & $304.44 \pm 72.57$ & $302.19 \pm 74.27$ & $347.65 \pm 85.71$ & 0.017 & 0.001 & 0.001 & 0.001 \\
\hline MCA left & $293.40 \pm 63.49$ & $289.11 \pm 63.45$ & $341.08 \pm 87.53$ & 0.007 & 0.001 & 0.001 & 0.001 \\
\hline Average & $261.73 \pm 112.01$ & $259.54 \pm 112.69$ & $295.78 \pm 135.67$ & 0.001 & 0.001 & 0.001 & 0.001 \\
\hline
\end{tabular}

hand, the attenuation of the bilateral vertebral arteries, basilar apex, and the bilateral M1 segments of the MCA were significantly higher in DLR than in FBP and Hybrid IR (all $p<$ $0.01)$.

The CNR was significantly higher in the DLR than in Hybrid IR and FBP for all patients $(p<0.001)$, resulting in an average improvement of $44.86 \%$ and $54.43 \%$ compared with Hybrid IR and FBP, respectively.

Similarly, the SNR was significantly higher in the DLR than in Hybrid IR and FBP, representing an average increase in the SNR by $46.22 \%$ (DLR vs Hybrid IR) and $55.37 \%$ (DLR vs FBP, $p<0.001)$.

The perimeter for coiled aneurysm and the surgical clip resulted in $3.93 \pm 0.11 \mathrm{~mm} ; 12.20 \pm 0.97 \mathrm{~mm}$ for FBP, 3.94 $\pm 0.10 \mathrm{~mm} ; 12.44 \pm 1.11 \mathrm{~mm}$ for Hybrid IR, and $3.82 \pm$ $0.99 \mathrm{~mm} ; 10.62 \pm 1.17 \mathrm{~mm}$ for DLR, respectively (Fig. 3D and Fig. 4D). The mean value of 10-90\% ERD for coiled aneurysm was $1.35 \pm 0.17$ with FBP, $1.47 \pm 0.88$ with Hybrid IR, and $1.04 \pm 0.22$ with DLR while it was $1.50 \pm 0.21$ with FBP, $1.62 \pm 0.16$ with Hybrid IR, and $0.85 \pm 0.27$ with DLR for surgical clip (Fig. 3E and Fig. 4E).

\subsection{Subjective analysis}

The subjective image quality scores of DLR were significantly higher than those of the Hybrid IR and FBP $(p<0.001)$ (Table 3). The interobserver agreement for subjective image analysis reached a $\mathrm{k}$ value of 0.77 , representing a good coefficient. Observer 1 scored all scans with DLR as acceptable image quality. According to observer 2, the image quality of DLR images was significantly higher than those of $\mathrm{Hy}$ brid IR and FBP images; however, no significant difference was observed between Hybrid IR and DLR. Fig. 5 demonstrates the increased number of cortical branches and welldemonstrated clinoid and supraclinoid segments of ICA. Furthermore, both observers judged the quality of the images to be highest with DLR than with FBP and Hybrid IR after assessing two sample images of patients with coiled aneurysms (Fig. 3) and surgically clipped (Fig. 4) by reducing blooming artifacts. For better visualization, the magnified images with red dashed outlines are illustrated in Figs. 3,4,6.

\section{Discussion}

We evaluated the image quality of brain CTA using different image reconstruction algorithms, i.e., FBP, Hybrid IR, and DLR. We found that compared to Hybrid IR and FBP, DLR significantly improved objective and subjective imag- 
Table 2. Results of CNR and SNR.

\begin{tabular}{|c|c|c|c|c|c|c|c|}
\hline & \multicolumn{3}{|c|}{ The mean value $\pm S D$} & \multicolumn{4}{|c|}{$p$-value } \\
\hline & FBP & Hybrid IR & DLR & $\begin{array}{c}\text { FBP vs Hybrid IR } \\
\text { vs DLR }\end{array}$ & FBP vs Hybrid IR & FBP vs DLR & Hybrid IR vs DLR \\
\hline \multicolumn{8}{|c|}{ SNR } \\
\hline ICA right & $18.14 \pm 5.47$ & $22.76 \pm 7.74$ & $33.03 \pm 12.25$ & 0.001 & 0.001 & 0.001 & 0.001 \\
\hline ICA left & $18.25 \pm 6.25$ & $22.14 \pm 9.59$ & $32.36 \pm 13.60$ & 0.001 & 0.001 & 0.001 & 0.001 \\
\hline VA right & $14.74 \pm 5.33$ & $17.52 \pm 6.05$ & $38.98 \pm 20.24$ & 0.001 & 0.001 & 0.001 & 0.001 \\
\hline VA left & $15.31 \pm 5.28$ & $18.53 \pm 6.78$ & $36.09 \pm 15.99$ & 0.001 & 0.001 & 0.001 & 0.001 \\
\hline BA apex & $13.98 \pm 5.30$ & $16.51 \pm 6.93$ & $32.85 \pm 15.26$ & 0.001 & 0.001 & 0.001 & 0.001 \\
\hline MCA right & $14.95 \pm 7.04$ & $18.05 \pm 8.71$ & $34.15 \pm 15.70$ & 0.001 & 0.001 & 0.001 & 0.001 \\
\hline MCA left & $13.99 \pm 3.47$ & $16.76 \pm 4.91$ & $32.53 \pm 13.52$ & 0.001 & 0.001 & 0.001 & 0.001 \\
\hline Average & $13.51 \pm 5.38$ & $16.28 \pm 7.12$ & $30.27 \pm 14.45$ & 0.001 & 0.001 & 0.001 & 0.001 \\
\hline \multicolumn{8}{|c|}{ CNR } \\
\hline ICA right & $15.99 \pm 5.21$ & $20.02 \pm 7.20$ & $29.27 \pm 11.60$ & 0.001 & 0.001 & 0.001 & 0.001 \\
\hline ICA left & $16.13 \pm 5.91$ & $19.54 \pm 9.04$ & $28.73 \pm 12.75$ & 0.001 & 0.001 & 0.001 & 0.001 \\
\hline VA right & $12.54 \pm 5.02$ & $14.84 \pm 5.62$ & $34.45 \pm 19.43$ & 0.001 & 0.001 & 0.001 & 0.001 \\
\hline VA left & $13.09 \pm 4.93$ & $15.80 \pm 6.29$ & $31.72 \pm 14.86$ & 0.001 & 0.001 & 0.001 & 0.001 \\
\hline BA apex & $11.85 \pm 4.76$ & $13.92 \pm 6.17$ & $28.80 \pm 14.11$ & 0.001 & 0.001 & 0.001 & 0.001 \\
\hline MCA right & $12.92 \pm 6.50$ & $15.49 \pm 7.89$ & $30.13 \pm 14.46$ & 0.001 & 0.001 & 0.001 & 0.001 \\
\hline MCA left & $12.03 \pm 3.21$ & $14.35 \pm 4.34$ & $28.83 \pm 12.65$ & 0.001 & 0.001 & 0.001 & 0.001 \\
\hline Average & $15.62 \pm 5.74$ & $18.90 \pm 7.68$ & $34.28 \pm 15.42$ & 0.001 & 0.001 & 0.001 & 0.001 \\
\hline
\end{tabular}

FBP, filtered back projection; Hybrid IR, hybrid iterative reconstruction; DLR, deep learning reconstruction; ICA, internal carotid artery; VA, vertebral artery; BA, basilar artery; MCA, middle cerebral artery; CNR, contrast-to-noise ratio; SNR, signal-to-noise ratio.

Table 3. The subjective image analysis scores.

\begin{tabular}{|c|c|c|c|c|c|c|c|}
\hline & \multirow{2}{*}{ FBP } & \multirow{2}{*}{ Hybrid IR } & \multirow{2}{*}{ DLR } & \multicolumn{4}{|c|}{$p$-value } \\
\hline & & & & FBP vs Hybrid IR vs DLR & FBP vs Hybrid IR & FBP vs DLR & Hybrid IR vs DLR \\
\hline \multicolumn{8}{|c|}{ Observer 1} \\
\hline Overall image quality & $2.14 \pm 0.35$ & $3.26 \pm 0.44$ & $3.46 \pm 0.51$ & 0.001 & 0.001 & 0.001 & 0.02 \\
\hline \multicolumn{8}{|c|}{ Observer 2} \\
\hline Overall image quality & $2.23 \pm 0.43$ & $3.30 \pm 0.46$ & $3.49 \pm 0.50$ & 0.001 & 0.001 & 0.001 & 0.074 \\
\hline
\end{tabular}

FBP, filtered back projection; Hybrid IR, hybrid iterative reconstruction; DLR, deep learning reconstruction.

ing quality by reducing image noise, blooming artifacts and improving SNR and CNR. Compared to Hybrid IR and FBP, DLR has the advantage of maintaining higher image quality while minimizing the cumulative radiation exposure with low tube voltage and current $[15,21]$. Recently, the fast speed reconstruction and radiation dose reduction have increased the interest in the use of DLR. Previous studies have investigated the importance of radiation dose reduction by DLR $[15,18]$. The vast learning capacities of the deep convolutional neural network allow for differentiation between the signal and the noise, and consequently, DLR reduces artifacts and noise separately from the signal. AiCE, a commercialized DLR used here, was trained to the target images of advanced model-based iterative reconstruction (MBIR); additionally, it overcomes the limitations of MBIR with fast reconstruction time and improved spatial resolution and image quality $[14,22]$. The reconstruction time is up to 3.5 images per sec for MBIR [15]. The reconstruction times by Hybrid IR and DLR were about 25 images per sec and 43 images per sec, which was similar to [15].

The DLR significantly reduced image noise and improved the attenuation for bilateral ICA segments, bilateral vertebral arteries, bilateral M1 segments of the MCA, and basilar apex, which led to significant improvement in the SNR and CNR. We found a $33.68 \%$ and $44.70 \%$ reduction in noise when using DLR compared to Hybrid IR and FBP. Additionally, the CNR was improved by $44.86 \%$ and $54.43 \%$ when using DLR compared to Hybrid IR and FBP, respectively. The DLR algorithm allowed an average increase in the SNR of $46.22 \%$ and 55.37\% compared to Hybrid IR and FBP. No study has investigated the use of DLR in brain CTA despite previous studies investigating DLR in other body sections $[13,15,16,18]$. 

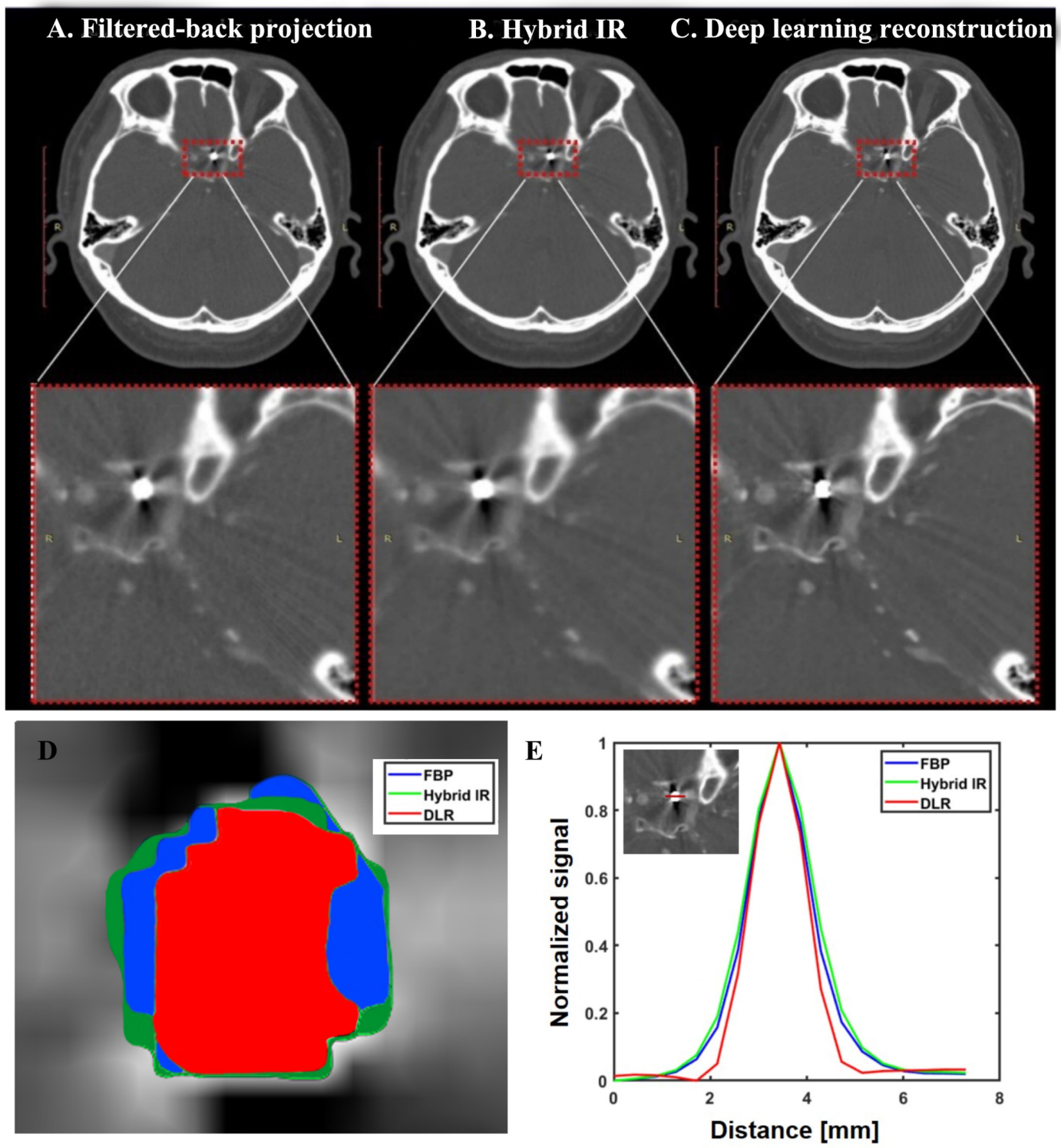

Fig. 3. Examples of brain CTA scan for a coiled aneurysm in the left paraclinoid internal carotid artery. The blooming artifact from coiling reduced in the deep learning reconstruction (DLR) (C) compared to filtered-back projection (FBP) (A) and hybrid iterative reconstruction (IR) (B). The coiling part can be seen on the magnified images with the red dashed outline for FBP, Hybrid IR, and DLR. Deep learning reconstruction was rated 3 scores, while the hybrid iterative and filtered-back projection were rated 2 by objective 2. The reduction blooming artifact and image sharpness were markedly improved in the deep learning reconstruction than FBP and Hybrid IR in terms of the perimeter (D) and normalized profile curve of 10-90\% edge rise distance (E).

Our results are consistent with previous studies investigating CT imaging of other organs [5, 13-17] despite the difference in location. The current and previous studies demonstrated the improved imaging quality when using DLR compared to Hybrid IR, MBIR, and FBP. We believe this improved image quality with DLR may be due to advanced MBIR images used for training DLR images. There was no significant difference in the attenuation of the right and left cavernous segments of the ICA among all image reconstruction methods. We consider that this phenomenon was caused by beam hardening artifacts in the ICA segments near the surrounding bone.
Kim et al. [16], investigating the commercialization of DLR, TrueFidelity ${ }^{T M}$, found improved image noise compared to Hybrid IR in Brain CT. Compared to previous work $[23,24]$, we assessed DLR's performance with more ROI's in various cerebral arteries. Lenfant et al. [15] reported that the use of AiCE allowed an approximately 25\% noise reduction and 20\% for both SNR and CNR improvement compared to AIDR 3D in pulmonary CTA. In our work, greater SNR and CNR improvements were observed in the posterior cranial fossa, including the basilar artery and the bilateral vertebral arteries. This indicates an advantage of DLR in diagnosing posterior fossa infarction, aneurysm, and dissection of 

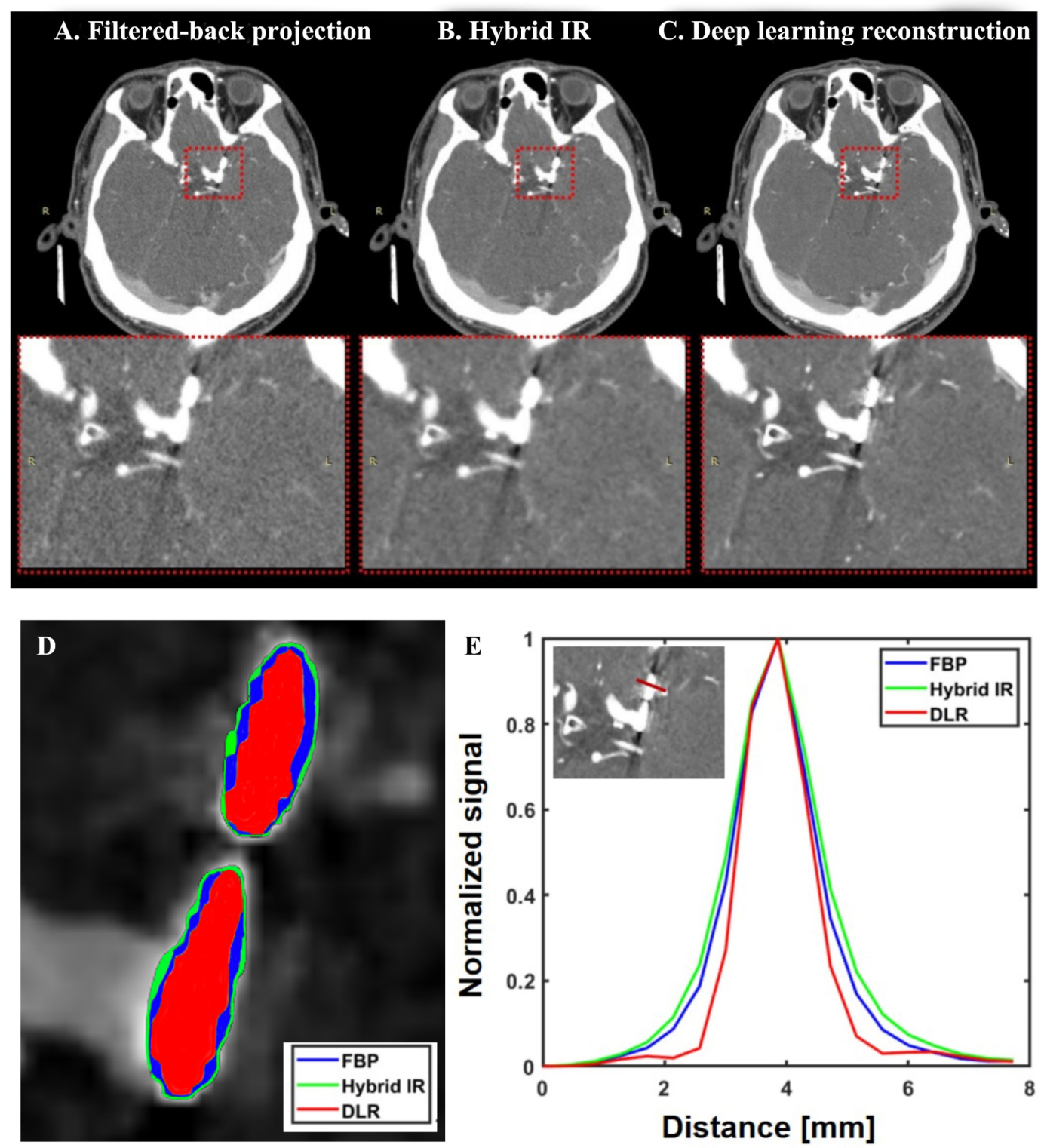

Fig. 4. Examples of brain CT angiographic scan for surgically clipped left paraclinoid aneurysms. The blooming artifact from surgically clips decreased in the deep learning reconstruction (DLR) (C) than hybrid iterative reconstruction (IR) (B) and filtered-back projection (FBP) (A). The surgical clip can be seen on the magnified images with the red dashed outline for FBP, Hybrid IR, and DLR. The subjective score of images qualified from two observers on deep learning reconstruction (score 4) was higher than hybrid iterative reconstruction (score 3) and filtered back projection (score 3). The use of DLR resulted in sharper images with reduction of blooming artifact compared to FBP and Hybrid IR when considering the analysis of perimeter (D) and normalized profile curve of $10-90 \%$ edge rise distance $(\mathrm{E})$.

these arteries. Increased diagnostic value of DLR is deemed to result from CNR, SNR, and noise reduction improvements. Further work is required to investigate the diagnostic accuracy of DLR in the posterior fossa.

Subjectively, improvements were observed in image quality when using DLR compared to FBP and Hybrid IR. According to observer 2, DLR images showed significantly better overall image quality than FBP and Hybrid IR; however, no significant difference was observed between Hybrid IR and DLR. The unfamiliar visual appearance of image features in DLR images may have affected this result, as reported previously $[16,25]$. The high $\mathrm{kVp}$ tube voltage and variable filter kernels are the most effective approach to overcome the blooming artifacts. Unfortunately, images at high $\mathrm{kVp}$ tube voltage suffernlmstringname at the cost of increased radiation dose. Therefore, the image reconstruction algorithms and kernel settings are viable options to reduce the stentrelated blooming artifact without exposure to high radiation doses. The reduction of blooming artifacts with DLR improved the visualization of intracranial vessels in the regions 


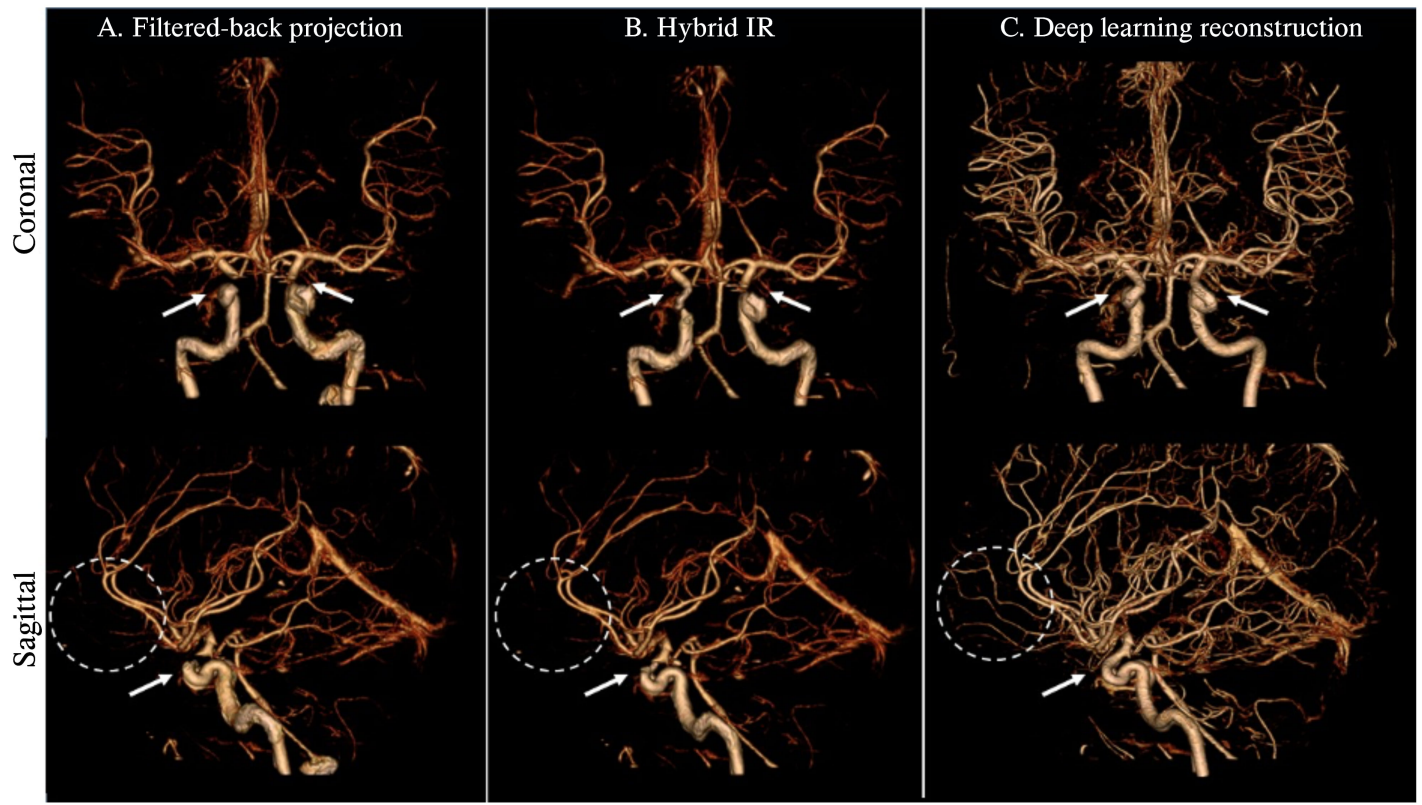

Fig. 5. Volume rendering of brain vessels extracted by bone removal from only CTA images. The volume-rendered image of brain CTA using deep learning reconstruction (C) shows an increased number of cortical branches and completeness of clinoid and supraclinoid segments of ICA (white arrows) compared to hybrid iterative (B) and filtered back projection (A). In addition, deep learning reconstruction allows more conspicuous visualization of the major terminal branches, including frontobasal and frontopolar arteries (dashed circle). At the same time, they are not seen in the hybrid iterative reconstruction (IR) and filtered back projection. Deep learning reconstruction provided significantly greater enhancement on small cortical branches of brain arteries, resulting in markedly improved vascular visualization. The images were views in the same window level and width.

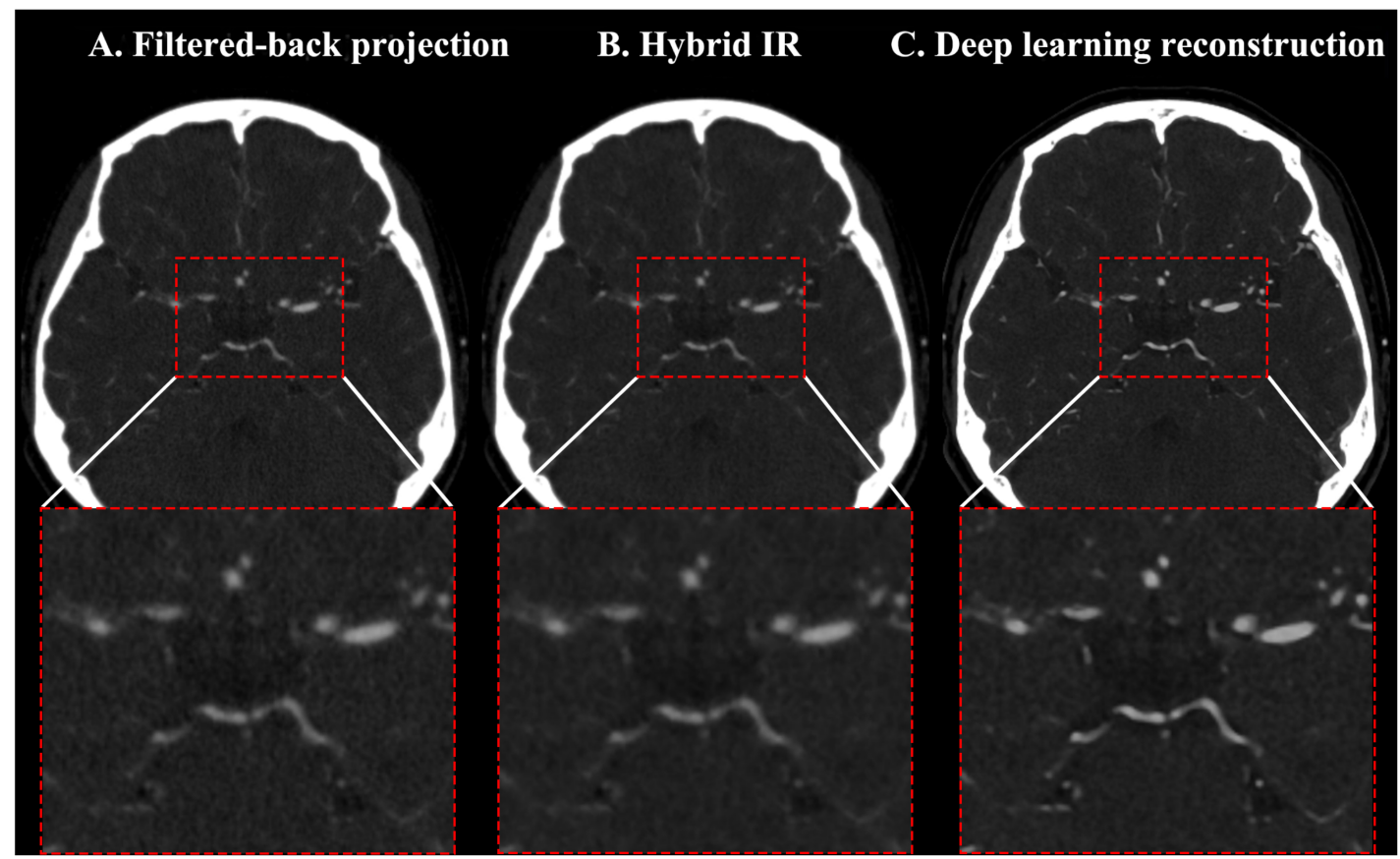

Fig. 6. Axial cut brain CTA images for visualization of intracranial vessel. Comparison of brain CTA between filtered-back projection (A), hybrid iterative reconstruction (IR) (B), and deep learning reconstruction (C) in the vessel visualization. The deep learning reconstructions (C) demonstrated a good vessel delineation wall with minimal image noise and sharp vessels compared to hybrid iterative (B) and filtered back projection (A) algorithms. The circle of Willis can be clear on the magnified images of the red dashed outlines. The image quality of the deep learning reconstruction was assessed by 4 scores, 3 scores for hybrid iterative reconstruction, and 2 scores for filtered-back projections by observer 1 . 
containing a surgical clip or coil. Therefore, DLR should have advantages in assessing the follow-up of aneurysms treated with flow diverters, stents, and clipping due to lower blooming artifacts. The use of DLR resulted in sharper images compared to FBP and Hybrid IR when considering analysis of 10-90\% ERD and perimeter. However, hybrid iterative reconstruction reduces the blooming artifact, which is strongly related to the kernel strength $[26,27]$. The result of 10-90\% ERD and perimeter of the coiled aneurysm and surgical clip in FBP was similar to Hybrid IR that may be affected by reconstruction kernels used in our paper (FC23 kernel for both FBP and Hybrid IR). Therefore, the optimized reconstruction kernel is required for the reduction of blooming artifacts.

As seen in Fig. 5, the cortical segments of large vessels and the clinoid and supraclinoid segments of the ICA were well-demonstrated. The vessel completeness was the highest in the DLR, which are impacted less by noise. The use of DLR has increased the spatial resolution [28, 29]. It has been shown to significantly enhance small cortical branches of brain arteries, resulting in markedly improved vascular visualization. In addition, increasing the volume or injection rate of contrast media could enhance the small peripheral vessels. Fig. 6 illustrates that the excellent vessel delineation wall and sharp vessels were more evident in DLR than in Hybrid IR and FBP. The improvement of the spatial resolution can decrease errors in the evaluation of small vessel and carotid stenosis. Thus, the deep learning image reconstruction may be especially helpful for delineating major terminal branches, e.g., frontobasal and frontopolar arteries, and accurately estimating the grade of carotid artery stenosis. Further studies need to be done to establish these qualities of DLR as benefits in detecting vascular abnormalities, including arteriovenous fistula, abnormalities of the collateral vessels, and distal aneurysms. The DLR resulted in sharper images and reduced the blooming artifact caused by surgical clips and coiled aneurysm compared to FBP and Hybrid IR without increasing the tube voltage that leads to high radiation dose for patients. The size of the surgical clip and coiled aneurysm appeared larger than its actual size due to blooming artifacts in the FBP and Hybrid IR than DLR. The visualization of intracranial vessels in the regions containing a surgical clip or coils could be evaluated accurately with DLR.

This work has several limitations. First, this was a retrospective study that only assessed a relatively small number of patients. Second, pathologic findings were not sufficiently studied. Third, we did not compare the radiation exposure in each reconstruction method in our study. In addition, image reconstructions in this study were provided by one CT.

Furthermore, the evaluation of image quality of brain CTA with deep learning reconstruction by other vendors is needed. Lastly, the analysis of 10-90\% ERD and perimeter in the surgical clip and coiled aneurysm was evaluated for only two patients and limited to coils and surgical clip types. Despite these limitations, improving vascular enhancement provides better visualization of small branches in the brain arteries without increasing the overall iodine dose delivery. As mentioned above, the image quality is improved in the deep learning reconstruction compared to filtered-back projection and hybrid iterative reconstruction.

\section{Conclusions}

The deep learning reconstruction method generally improves the image quality of brain CTA in terms of objective measurement and subjective grading compared with filtered back projection and hybrid iterative reconstruction methods. Especially, deep learning reconstruction is deemed advantageous for better depicting small vessels than FBP and Hybrid IR.

\section{Abbreviations}

CTA, Computed Tomography Angiography; IR, Iterative Reconstruction; MBIR, Model-Based Iterative Reconstruction; DLR, Deep Learning Reconstruction; CNR, Contrastto-noise ratio; FBP, Filtered-Back Projection; AiCE, Advanced Intelligent Clear-IQ Engine; SNR, Signal-to-noise ratio; ROI, Regions of interest; ICA, Internal carotid artery; MCA, Middle cerebral artery.

\section{Author contributions}

CO, JKR and SKK designed the research study. CO, HJS, DHH and SKK performed the research. HJS, DHH and JWS provided help and advice on the research. CO and JKR wrote the manuscript. CO and JKR analyzed the data. All authors contributed to editorial changes in the manuscript. All authors read and approved the final manuscript.

\section{Ethics approval and consent to participate}

The institutional review board of the Inje University Seoul Paik Hospital approved this retrospective study, IRB File No. 2021-06-008.

\section{Acknowledgment}

Not applicable.

\section{Funding}

This work was supported by the Korea Medical Device Development Fund grant funded by the Korea government (the Ministry of Science and ICT, the Ministry of Trade, Industry and Energy, the Ministry of Health \& Welfare, the Ministry of Food and Drug Safety) (Project number: 1711139017).

\section{Conflict of interest}

The co-authors (Jae-Kyun Ryu, Seonkyu Kim) are employees of Canon Medical Systems Korea, Seoul, Korea that is the subsidiary in Korea of Canon Medical Systems Corporation, Otawara-si, Japan. The authors declare the paper has no conflicts of interest with the company. 


\section{References}

[1] Lian K, Bharatha A, Aviv RI, Symons SP. Interpretation Errors in CT Angiography of the Head and Neck and the Benefit of Double Reading. American Journal of Neuroradiology. 2011; 32: 21322135.

[2] Matsumoto M, Sato M, Nakano M, Endo Y, Watanabe Y, Sasaki $\mathrm{T}$, et al. Three-dimensional computerized tomography angiography-guided surgery of acutely ruptured cerebral aneurysms. Journal of Neurosurgery. 2001; 94: 718-727.

[3] Stiller W. Basics of iterative reconstruction methods in computed tomography: a vendor-independent overview. European Journal of Radiology. 2018; 109: 147-154.

[4] Katsura M, Sato J, Akahane M, Matsuda I, Ishida M, Yasaka K, et al. Comparison of pure and hybrid iterative reconstruction techniques with conventional filtered back projection: Image quality assessment in the cervicothoracic region. European Journal of Radiology. 2013; 82: 356-360.

[5] Hamamura T, Hayashida Y, Takeshita Y, Sugimoto K, Ueda I, Futatsuya $\mathrm{K}$, et al. The usefulness of full-iterative reconstruction algorithm for the visualization of cystic artery on CT angiography. Japanese Journal of Radiology. 2019; 37: 526-533.

[6] Gaddikeri S, Andre JB, Benjert J, Hippe DS, Anzai Y. Impact of Model-Based Iterative Reconstruction on Image Quality of Contrast-Enhanced Neck CT. American Journal of Neuroradiology. 2015; 36: 391-396.

[7] Racine D, Becce F, Viry A, Monnin P, Thomsen B, Verdun FR, et al. Task-based characterization of a deep learning image reconstruction and comparison with filtered back-projection and a partial model-based iterative reconstruction in abdominal CT: A phantom study. Physica Medica. 2020; 76: 28-37.

[8] Benz DC, Benetos G, Rampidis G, von Felten E, Bakula A, Sus$\operatorname{tar} A$, et al. Validation of deep-learning image reconstruction for coronary computed tomography angiography: Impact on noise, image quality and diagnostic accuracy. Journal of Cardiovascular Computed Tomography. 2020; 14: 444-451.

[9] Marin D, Nelson RC, Schindera ST, Richard S, Youngblood RS, Yoshizumi TT, et al. Low-Tube-Voltage, High-Tube-Current Multidetector Abdominal CT: Improved Image Quality and Decreased Radiation Dose with Adaptive Statistical Iterative Reconstruction Algorithm - Initial Clinical Experience. Radiology. 2010; 254: 145-153.

[10] Singh R, Digumarthy SR, Muse VV, Kambadakone AR, Blake MA, Tabari A, et al. Image Quality and Lesion Detection on Deep Learning Reconstruction and Iterative Reconstruction of Submillisievert Chest and Abdominal CT. American Journal of Roentgenology. 2020; 214: 566-573.

[11] Solomon J, Lyu P, Marin D, Samei E. Noise and spatial resolution properties of a commercially available deep learning-based CT reconstruction algorithm. Medical Physics. 2020; 47: 3961-3971.

[12] Zhang Z, Seeram E. The use of artificial intelligence in computed tomography image reconstruction - a literature review. Journal of Medical Imaging and Radiation Sciences. 2020; 51: 671-677.

[13] Akagi M, Nakamura Y, Higaki T, Narita K, Honda Y, Zhou J, et al. Deep learning reconstruction improves image quality of abdominal ultra-high-resolution CT. European Radiology. 2019; 29: 6163-6171.

[14] Tatsugami F, Higaki T, Nakamura Y, Yu Z, Zhou J, Lu Y, et al. Deep learning-based image restoration algorithm for coronary CT angiography. European Radiology. 2019; 29: 5322-5329.

[15] Lenfant M, Chevallier O, Comby PO, Secco G, Haioun K, Ricolfi F, et al. Deep Learning Versus Iterative Reconstruction for CT Pulmonary Angiography in the Emergency Setting: Improved Image Quality and Reduced Radiation Dose. Diagnostics. 2020; 10: 558.
[16] Kim I, Kang H, Yoon HJ, Chung BM, Shin N. Deep learningbased image reconstruction for brain CT: improved image quality compared with adaptive statistical iterative reconstruction-Veo (ASIR-V). Neuroradiology. 2021; 63: 905-912.

[17] Kim JH, Yoon HJ, Lee E, Kim I, Cha YK, Bak SH. Validation of Deep-Learning Image Reconstruction for Low-Dose Chest Computed Tomography Scan: Emphasis on Image Quality and Noise. Korean Journal of Radiology. 2021; 22: 131-138.

[18] Bernard A, Comby PO, Lemogne B, Haioun K, Ricolfi F, Chevallier $\mathrm{O}$, et al. Deep learning reconstruction versus iterative reconstruction for cardiac CT angiography in a stroke imaging protocol: reduced radiation dose and improved image quality. Quantitative Imaging in Medicine and Surgery. 2021; 11: 392-401.

[19] Tatsugami F, Higaki T, Sakane H, Fukumoto W, Kaichi Y, Iida M, et al. Coronary Artery Stent Evaluation with Model-based Iterative Reconstruction at Coronary CT Angiography. Academic Radiology. 2017; 24: 975-981.

[20] den Harder AM, Willemink MJ, van Doormaal PJ, Wessels FJ, Lock MTWT, Schilham AMR, et al. Radiation dose reduction for CT assessment of urolithiasis using iterative reconstruction: a prospective intra-individual study. European Radiology. 2018; 28: $143-150$.

[21] Shin YJ, Chang W, Ye JC, Kang E, Oh DY, Lee YJ, et al. Low-Dose Abdominal CT Using a Deep Learning-Based Denoising Algorithm: a Comparison with CT Reconstructed with Filtered Back Projection or Iterative Reconstruction Algorithm. Korean Journal of Radiology. 2020; 21: 356-364.

[22] Willemink MJ, Noël PB. The evolution of image reconstruction for CT-from filtered back projection to artificial intelligence. European Radiology. 2019; 29: 2185-2195.

[23] Löve A, Siemund R, Höglund P, Ramgren B, Undrén P, BjörkmanBurtscher IM. Hybrid iterative reconstruction algorithm improves image quality in craniocervical CT angiography. American Journal of Roentgenology. 2014; 201: W861-W866.

[24] Niesten JM, van der Schaaf IC, Vos PC, Willemink MJ, Velthuis BK. Improving head and neck CTA with hybrid and model-based iterative reconstruction techniques. Clinical Radiology. 2015; 70: 1252-1259.

[25] Geyer LL, Schoepf UJ, Meinel FG, Nance JW, Bastarrika G, Leipsic JA, et al. State of the Art: Iterative CT Reconstruction Techniques. Radiology. 2015; 276: 339-357.

[26] Oda S, Utsunomiya D, Funama Y, Takaoka H, Katahira K, Honda $\mathrm{K}$, et al. Improved coronary in-stent visualization using a combined high-resolution kernel and a hybrid iterative reconstruction technique at 256-slice cardiac CT-Pilot study. European Journal of Radiology. 2013; 82: 288-295.

[27] Hong SG, Kang E, Park JH, Choi WJ, Lee K, Kwon HJ, et al. Effect of Hybrid Kernel and Iterative Reconstruction on Objective and Subjective Analysis of Lung Nodule Calcification in Low-Dose Chest CT. Korean Journal of Radiology. 2018; 19: 888-896.

[28] Higaki T, Nakamura Y, Zhou J, Yu Z, Nemoto T, Tatsugami F, et al. Deep Learning Reconstruction at CT: Phantom Study of the Image Characteristics. Academic Radiology. 2020; 27: 82-87.

[29] Urikura A, Ichikawa K, Hara T, Nishimaru E, Nakaya Y. Spatial resolution measurement for iterative reconstruction by use of image-averaging techniques in computed tomography. Radiological Physics and Technology. 2014; 7: 358-366. 